\title{
LA AUTONOMÍA DEL DERECHO COMUNITARIO ANDINO Y SU RELACIÓN CON EL DERECHO INTERNACIONAL
}

\section{AUTONOMIA DO DIREITO COMUNITÁRIO ANDINO E SUA RELAÇÃO COM O DIREITO INTERNACIONAL}

Carolina Lourdes Rodríguez Aguilera

Resumen: La propuesta de indagar acerca de la autonomía del derecho comunitario andino, a partir del sistema de solución de controversias de la Comunidad Andina es un tema de mucho interés, que refleja una realidad compleja en el escenario comercial internacional, como son las interacciones que se están dando entre iniciativas regionales y multilaterales en materia comercial, que son objeto de profundos debates. Este trabajo se centrará en el estudio de casos, en un área específica como las salvaguardias.

Resumo: A proposta para saber sobre a autonomia do direito comunitário andino, a partir do sistema de solução de controvérsias Comunidade Andina é um assunto de grande interesse, o que reflete uma realidade complexa na arena do comércio internacional, tais interações são dando iniciativas regionais e multilaterais sobre questões comerciais, que são objeto de intenso debate. Este trabalho vai se concentrar em estudos de caso em uma área específica, tais como salvaguardas.

Palabras clave: Integración económica regional, Solución de controversias, Ordenamiento jurídico comunitario andino, Restricciones y gravámenes, Salvaguardias

Palavras-chave: Integração económica regional, Resolução de conflitos, Comunidade andina sistema legal, Restrições e gravames, Salvaguardas

\section{INTRODUCCIÓN}

La interdependencia asimétrica ${ }^{1}$ que caracteriza a los países demanda mayores esfuerzos y necesidad de coordinación. Desarrollar

\footnotetext{
* Profesora adscrita al Departamento de Ciencias Sociales de la Universidad Simón Bolívar E-mail: rodriguezcarol@usb.ve

1 KEOHANE, Robert O., y NYE Joseph. Poder e Interdependencia. Buenos Aires: Grupo Editor Latinoamericano. Colección Estudios Internacionales, 1988.
} 
mecanismos de entendimientos y de interpretación de los acuerdos, así como, profundizar en el conocimiento e implementación de los diversos sistemas de solución de controversias, pueden resultar relevantes para el afianzamiento del bloque regional y su inserción en el escenario multilateral, y tiene importancia para atraer inversiones, tanto nacionales como extranjeras, y contribuye a impulsar el desarrollo económico de un país o de la región.

Los acuerdos de integración regional, pueden favorecer la inclusión internacional de los países, mejorando la competitividad de sus exportaciones. Incluso hay quienes sostienen ${ }^{2}$ que la integración económica regional es uno de los medios para que los países puedan acelerar su proceso de desarrollo económico y social, y promover un proceso que conduzca al establecimiento de un mercado común regional. Sin embargo, estas metas no están exentas de la influencia del contexto en que se desarrolla el proceso, la infraestructura existente y las capacidades y ventajas de los países participantes, así como, depende en gran medida de la institucionalidad existente, particularmente, del sistema normativo y jurisdiccional, que garantice y controle el cumplimiento de las obligaciones y derechos pactados.

En ese contexto, cobraría valor el patrimonio del ordenamiento jurídico de la Comunidad Andina (CAN), como un elemento de seguridad jurídica de respeto de las normas, que propicie la inversión y favorezca el libre intercambio de bienes y servicios.

Para cumplir con este propósito, se hace indispensable ilustrar esta situación a partir de la realidad misma donde se generan este tipo de interacciones, a través del estudio de dos controversias entre Países Miembros de la Comunidad Andina donde estuvieron involucradas una norma regional andina (salvaguardia comercial andina prevista en el Acuerdo de Cartagena), y una norma multilateral de la Organización Mundial del Comercio (medida de salvaguardia estipulada en el Acuerdo sobre Salvaguardias de la OMC).

En ese orden, se considera importante presentar y analizar cuáles han sido los criterios de interpretación desarrollados por el sistema de solución de controversias andino, sobre la relación entre el ordenamiento jurídico comunitario andino y la normativa multilateral de la Organización Mundial del Comercio (OMC).

A la luz de las consideraciones anteriores, puede observarse que este trabajo parte de un enfoque jurídico, como es el sistema de solución de controversias andino.

2 SUNKEL, Osvaldo. "Reflexiones sobre América Latina y el Caribe. Desarrollo e Integración Regional". Revista de la CEPAL. 1998, número extraordinario; FERRER Aldo. "Desarrollo e Integración: Desafíos y Oportunidades del MERCOSUR". Revista Vértice. 2007, n², p. 7-9; NARANJO Fernando. "Política Exterior e Integración. Oportunidades y Desafíos". Cuadernos Integración en América Latina. Santiago de Chile: CEPAL/Fundación Carolina/ FLACSO, 2006. 
El derecho comunitario andino aparece como un conjunto de normas con características especiales- supremacía-, y un sistema propio de solución de controversias que es objeto de control y vigilancia por parte de dos instituciones, la Secretaría General de la Comunidad Andina y el Tribunal de Justicia Andino, que han contribuido a la interpretación uniforme del derecho comunitario andino, y a generar estabilidad y seguridad jurídica, en el sentido de los derechos y obligaciones recogidos en los tratados fundamentales.

\section{EL ORDENAMIENTO JURÍDICO DE LA COMUNIDAD ANDINA}

Cuando se pacta un acuerdo de integración, se establece un fin a alcanzar, y para la consecución del mismo, se espera que los países comprometidos realicen una serie de actividades (obligación de hacer) o dejen de realizar aquellas actuaciones contrarias al objetivo a alcanzar (obligación de no hacer) y preserven una conducta acorde con el compromiso asumido ${ }^{3}$.En la Comunidad Andina, los Países Miembros pactaron la conformación de un mercado común, como un instrumento para el intercambio intracomunitario y el desarrollo de la población.

La Declaración de Bogotá del 16 de agosto de $1966^{4}$ marca el comienzo del proyecto de integración andino, con la participación de los Presidentes de Colombia (Carlos Lleras Restrepo), Chile (Eduardo Frei) y Venezuela (Raúl Leoni), y el delegado del gobierno de Ecuador (Galo Plaza) y de Perú (Fernando Schwalb). El Gobierno de Bolivia se incorporó luego a este grupo y participó en la Comisión que se creó para estudiar la iniciativa de formar un grupo de integración subregional andino. De las deliberaciones realizadas por la Comisión se produjo como resultado la suscripción del Acuerdo de Integración Subregional Andino, el 26 de mayo de 1969, conocido como Acuerdo de Cartagena,

\footnotetext{
3 Artículo 4 del Tratado de Creación del Tribunal de Justicia de la Comunidad Andina: Los Países Miembros están obligados a adoptar las medidas que sean necesarias para asegurar el cumplimiento de las normas que conforman el ordenamiento jurídico de la Comunidad Andina. Se comprometen, asimismo, a no adoptar ni emplear medida alguna que sea contraria a dichas normas o que de algún modo obstaculice su aplicación.

Se recomienda ver: Acuerdo de Cartagena; así como: MONSANTO, Alberto. "CANMERCOSUR: Esquemas institucionales comparados". En: UNIVERSIDAD NACIONAL DEL ROSARIO, CÁTEDRA INTERNACIONAL ANDRÉS BELLO. Integración y Cooperación Atlántico Pacífico. Rosario: Editorial Universidad Nacional de Rosario, 2002; SESELOVSKY, Ernesto, et al. "Necesidad de armonización de las políticas macroeconómicas en los procesos de integración regional”. En: UNIVERSIDAD NACIONAL DEL ROSARIO, CATEDRA INTERNACIONAL ANDRÉS BELLO (ed.). Integración y Cooperación Atlántico-Pacífico. Rosario: Editorial Universidad Nacional de Rosario, 2002; LEVI, Michel. La Comunidad Andina y el referente de la Unión Europea: Una visión temporal sobre la aplicación de los modelos de integración. La Unión Europea y la Integración regional. Perspectivas comparadas y lecciones para las Américas. Buenos Aires: CARI, Eduntref, University of Miami, 2005, p. 267-295.

4 MALDONADO LIRA, Héctor. 30 años de integración andina. Balances y perspectivas. Lima: Secretaría General de la Comunidad Andina, 1999, p. 34.
} 
por los gobiernos de Bolivia, Colombia, Chile, Ecuador y Perú, con el objetivo de crear un mercado común 5 .

En un primer momento, el Acuerdo de Cartagena no contaba con un sistema de solución de controversias propio, sino que se regía por el procedimiento de solución de controversias pautado en el Tratado de Montevideo de 1960, que básicamente disponía, la negociación, la conciliación y el arbitraje.

Sin embargo, el surgimiento del Grupo Andino ha estado acompañado de un proceso de desarrollo paulatino de sus instituciones ${ }^{6}$, vale destacar la aprobación del Tratado de Creación del Tribunal de Justicia del Acuerdo de Cartagena, el 28 de mayo de 1979. Esta institución significó un punto importante en el desarrollo de la integración andina que pasó a tener su propio órgano de solución de controversias, que serviría además, como órgano de control de la legalidad del ordenamiento jurídico comunitario andino, y de la interpretación uniforme de sus normas, y a la vez, contribuiría a la generación de una doctrina jurídica andina.

Con el Acuerdo de Cartagena que constituye la Carta fundacional y los Tratadosy Protocolos subsiguientes ${ }^{7}$, el modelo de integración dela Comunidad Andina se fue conformando como un grupo con vigencia indefinida.

La etapa siguiente a destacar es el Protocolo Modificatorio del Tratado de Creación del Tribunal de Justicia de la Comunidad Andina, aprobado en Cochabamba, Bolivia el 28 de mayo de $1996^{9}$. A través de este instrumento jurídico, los países firmantes acordaron aumentar las competencias del Tribunal, entre ellas, es preciso señalar, el procedimiento de arbitraje, recurso por omisión o inactividad de los órganos e instituciones de la Comunidad Andina, y la jurisdicción laboral para los funcionarios del sistema de integración andino.

Por su parte, es pertinente indicar que el Protocolo de Trujillo, de 10 de marzo de 1996, en vigencia desde el 2 de junio de 1997, incorporó reformas en la estructura de la organización de integración andina que pasó a denominarse Comunidad Andina. Consecuentemente, se aprobó el Protocolo de Sucre (Modificatorio del Acuerdo de Cartagena), en Quito, el 25 de junio de 1997. Este Protocolo encargó a la Comisión de la Comunidad Andina la aprobación de una Decisión que consagrara

\footnotetext{
5 Venezuela ingresa el 13 de febrero de 1973. Por otra parte, Chile se retiró del Acuerdo de Cartagena en 1976, tras el derrocamiento del Gobierno del Presidente Salvador Allende.

6 El 25 de octubre de ese mismo año, se suscribe también el Tratado Constitutivo del Parlamento Andino.

7 Protocolo de Quito de 1987; artículo 30 del Tratado de Creación del Tribunal de Justicia del Acuerdo de Cartagena; artículo 3 literal d) y 134 del Acuerdo de Cartagena (Decisión 563).

8 Artículo 134 del Acuerdo de Cartagena: Este Acuerdo no podrá ser suscrito con reservas y permanecerá en vigencia por tiempo indefinido.

9 El Protocolo Modificatorio entró en vigencia el 25 de agosto de 1999, fecha en la cual quedaron depositados todos los instrumentos de ratificación en la Secretaría General de la Comunidad Andina. Este instrumento jurídico modificó el nombre del Tribunal -antes Tribunal de Justicia del Acuerdo de Cartagena- que pasó a llamarse Tribunal de Justicia de la Comunidad Andina.
} 
las modificaciones introducidas, y en ese orden, surge la Decisión 563 de la Comisión que codificó el Acuerdo de Cartagena ${ }^{10}$.

Puede observarse en el Preámbulo del Acuerdo de Cartagena una referencia a la Declaración de Bogotá, y los Países Miembros plantean la integración como un "mandato histórico, político, económico, social y cultural" que a través de la consecución de ciertos objetivos pretenden un mejoramiento en el nivel de vida de los habitantes. En ese sentido, el Acuerdo de Cartagena prevé como objetivos ${ }^{11}$ :

Promover el desarrollo equilibrado y armónico de los Países Miembros en condiciones de equidad, mediante la integración y la cooperación económica y social; acelerar su crecimiento y la generación de ocupación; facilitar su participación en el proceso de integración regional, con miras a la formación gradual de un mercado común latinoamericano.

\section{El Acuerdo continúa agregando la necesidad de:}

Propender a disminuir la vulnerabilidad externa y mejorar la posición de los Países Miembros en el contexto económico internacional; fortalecer la solidaridad subregional y reducir las diferencias de desarrollo existentes entre los Países Miembros ${ }^{12}$.

También es preciso señalar que, la Comunidad Andina es concebida como una organización subregional con personería o personalidad jurídica internacional ${ }^{13}$, actualmente está conformada por Bolivia, Colombia, Ecuador y Perú ${ }^{14}$ y por los órganos e instituciones del Sistema Andino de Integración (SAI) ${ }^{15}$. Asimismo, participan como países asociados Chile, Argentina, Brasil, Paraguay y Uruguay, y como observadores, México y Panamá.

El ordenamiento jurídico de la Comunidad Andina presenta características particulares, conviene destacar, la aplicación directa e inmediata y la preeminencia o supremacía.

10 Sustituye a la Decisión 406 de la Comisión.

11 Artículo 1 del Acuerdo de Cartagena, (Decisión 563 de la Comisión de la Comunidad Andina, que contiene la Codificación del Acuerdo de Cartagena).

12 Ibídem.

13 Artículo 48 Ibídem.

14 Venezuela denunció el Acuerdo de Cartagena en abril de 2006 con base en el artículo 135 del mismo Acuerdo, por lo que cesaron para ese país los derechos y obligaciones derivados de su condición de Miembro, con excepción de las ventajas recibidas y otorgadas de conformidad con el Programa de Liberación de la Subregión, las cuales permanecerán en vigencia por un plazo de cinco años a partir de la denuncia. Es importante señalar que hasta que se cumpla ese lapso o se concrete el retiro definitivo, Venezuela debe preservar el trato nacional; el libre comercio andino; otorgar el beneficio de nación más favorecida. Para revisar con más detalle este punto véase Decisión 641 de la Comisión de la CAN.

15 Artículo 5 del Acuerdo de Cartagena. 


\subsection{Aplicación inmediata y efecto directo}

Esta cualidad implica que las Decisiones y Resoluciones de la CAN son de obligatorio cumplimiento para los Países Miembros, así como para los órganos e instituciones del SAI, desde la fecha en que son aprobadas por el Consejo Andino de Ministros de Relaciones Exteriores o por la Comisión de la Comunidad Andina ${ }^{16}$.

Es propicio destacar que el Tratado de Creación del Tribunal de Justicia de la Comunidad Andina precisa que las Decisiones y Resoluciones serán directamente aplicables en los Países Miembros a partir de la fecha de su publicación en la Gaceta Oficial del Acuerdo de Cartagena $^{17}$, a menos que las mismas señalen una fecha posterior ${ }^{18}$. $Y$ agrega dicho Tratado que, en ocasiones cuando su texto así lo disponga, las Decisiones requerirán de incorporación al derecho interno, mediante acto expreso en el cual se indicará la fecha de su entrada en vigor en cada País Miembro.

Esta categoría de aplicación directa implica que no es necesario un proceso de intermediación o validación por parte de los organismos competentes de los Países Miembros de la Comunidad Andina. Así por ejemplo, en el caso de las sentencias y laudos del Tribunal y los pronunciamientos -Resoluciones- de la Secretaría General no se requiere de homologación o exequátur en ninguno de los Países Miembros ${ }^{19}$.

De esta forma la norma comunitaria andina derivada entraría en vigencia para todos simultáneamente y de manera inmediata. Se distingue a la norma comunitaria andina derivada de las normas originarias, porque en el caso de las normas andinas primarias, como los Tratados constitutivos o fundacionales, estos entrarían en vigencia de conformidad con lo pautado en el Tratado, y con el procedimiento nacional en materia de aprobación de tratados internacionales ${ }^{20}$.

16 Artículo 2 del Tratado de Creación del Tribunal de Justicia de la Comunidad Andina. 17 Vale señalar que corresponde a la Secretaría General de la Comunidad Andina, editar la Gaceta la Gaceta Oficial del Acuerdo de Cartagena, véase en: Artículo 30 literal m) del Acuerdo de Cartagena; y, artículo 43 del Tratado de Creación del Tribunal de Justicia de la Comunidad Andina. En la Gaceta Oficial del Acuerdo de Cartagena se publicarán las Decisiones del Consejo Andino de Ministros de Relaciones Exteriores, de la Comisión de la Comunidad Andina, los Convenios, las Resoluciones y Dictámenes de la Secretaría General y las sentencias del Tribunal. Así también, el Secretario General podrá disponer, excepcionalmente, la publicación de otros actos jurídicos, siempre que éstos tengan carácter general y su conocimiento sea de interés para la Comunidad Andina.

18 Artículo 3 del Tratado de Creación del Tribunal de Justicia de la Comunidad Andina.

19 Artículo 41 Ibídem.

20 Así por ejemplo, el Tratado de Creación del Tribunal de Justicia de la Comunidad Andina estipuló que:

El presente Protocolo Modificatorio entrará en vigencia cuando todos los Países Miembros que lo suscriban hayan depositado el respectivo instrumento de ratificación en la Secretaría General de la Comunidad Andina y haya entrado en vigencia el Protocolo Modificatorio del Acuerdo de Integración Subregional Andino (Acuerdo de Cartagena) aprobado en Trujillo, Perú, el 10 de marzo de 1996. Por su parte, el artículo 134 del Acuerdo de Cartagena dispuso 


\subsection{Preeminencia o supremacía}

Las normas comunitarias andinas se identifican por estar dotadas de supremacía o preeminencia, esto significa que prevalecen en las materias de su competencia, sobre las normas del ordenamiento jurídico interno de los Estados ${ }^{21}$. Esta peculiaridad ha sido explicada por el Tribunal de Justicia de la Comunidad Andina, en los siguientes términos:

El ordenamiento jurídico de la integración andina prevalece en su aplicación sobre las normas internas o nacionales, por ser característica esencial del Derecho Comunitario, como requisito básico para la construcción integracionista ${ }^{22}$.

En ese orden, según la interpretación que ha hecho el Tribunal de Justicia andino ha precisado que:

En cuanto al efecto de las normas de la integración sobre las normas nacionales, señalan la doctrina y la jurisprudencia que, en caso de conflicto, la regla interna queda desplazada por la comunitaria, la cual se aplica preferentemente ya que la competencia en el caso le corresponde a la comunidad. En otros términos, la norma interna resulta inaplicable, en beneficio de la norma comunitaria(...).No se trata propiamente de que la norma comunitaria posterior derogue a la norma nacional preexistente, al igual que ocurre en el plano del derecho interno, puesto que son dos ordenamientos jurídicos distintos, autónomos y separados, que adoptan dentro de sus propias competencias formas peculiares de crear y extinguir el derecho, que por supuesto no son intercambiables(...). Frente a la norma comunitaria, los Estados Miembros(...) no pueden formular reservas ni desistir unilateralmente de aplicarla, ni pueden escudarse en disposiciones vigentes o en prácticas usuales de su orden interno para justificar el incumplimiento o la alteración de obligaciones resultantes del derecho comunitario ${ }^{23}$.

El compromiso asumido por los países andinos supone una

que: El presente Acuerdo entrará en vigencia cuando todos los Países Miembros que lo suscriben hayan depositado el respectivo instrumento de ratificación en la Secretaría General de la Comunidad Andina.

Este Acuerdo no podrá ser suscrito con reservas y permanecerá en vigencia por tiempo indefinido.

21 Tribunal de Justicia de la Comunidad Andina, sentencia 1-IP-96 (Interpretación prejudicial): La transferencia del poder regulador en determinadas materias de interés común de los Estados, a un órgano comunitario para alcanzar las metas de la integración, produce el fenómeno de redistribución de funciones y atribuciones entre los órganos comunitarios y los Países Miembros.

22 Tribunal de Justicia de la Comunidad Andina, sentencia del 3 de diciembre de 1987.

23 Tribunal de Justicia de la Comunidad Andina, proceso de interpretación prejudicial 2 IP 88. 
conducta en concordancia con las obligaciones y derechos acordados, en ese sentido, se espera que respeten los principios comunitarios. Ahora bien, según la interpretación que ha dado el Tribunal de Justicia de la Comunidad Andina, sobre la relación de la norma andina respecto a las normas internacionales, ha considerado que:

En lo que concierne a la relación propiamente dicha entre el ordenamiento comunitario y el internacional, (...) este constituye una de las fuentes de aquel, pero que de ello no se deriva que la Comunidad quede obligada por el citado ordenamiento ${ }^{24}$.

De acuerdo a lo que se expresa en esta sentencia del Tribunal Andino, por una parte, ese organismo reconoce que el derecho internacional es fuente del derecho comunitario y están vinculados, aunque parece querer destacar una autonomía del derecho comunitario en la aplicación para los Países Miembros. Así lo ha expuesto el Tribunal Andino:

El ordenamiento jurídico andino es autónomo y la aplicación de las normas comunitarias que lo conforman no depende de la de otros ordenamientos internacionales, ni debe sujetarse a que guarden compatibilidad o conformidad con ellas. Cosa bien diferente es la de que, para que este ordenamiento se acompase con el de otras esferas $\mathrm{u}$ organizaciones internacionales o mundiales, el legislador andino expida normas que acojan dentro de su ordenamiento principios y regulaciones idénticas o semejantes a las de aquellas...Además, el derecho comunitario andino, fuera de constituir un ordenamiento jurídico autónomo, independiente, con su propio sistema de producción, ejecución y aplicación normativa, posee los atributos, derivados de su propia naturaleza, conocidos como aplicabilidad inmediata, efecto directo y primacía ${ }^{25}$.

Ahora, el Tribunal Andino también se ha referido a la relación de los países con esquemas regionales y multilaterales, y al respecto ha señalado que:

En particular, en lo que concierne al doble vínculo de los Países Miembros con el ordenamiento jurídico de la Comunidad y con el Acuerdo de Marrakech por el que se estableció la Organización Mundial del Comercio, el criterio del Tribunal ha sido que:

La circunstancia de que los Países Miembros de la Comunidad Andina pertenezcan a su vez a la Organización Mundial del Comercio no los

24 Tribunal de Justicia de la Comunidad Andina, sentencia 1-IP-96 (Interpretación prejudicial). 25 Tribunal de Justicia de la Comunidad Andina, sentencias 89-AI-2000 (acción de incumplimiento), 10 AN 2000 y 61 AN 2000 (Acción de nulidad). 
exime de obedecer las normas comunitarias andinas so pretexto de que se está cumpliendo con las de dicha organización o que se pretende cumplir con los compromisos adquiridos con ella ${ }^{26}$.

En este pronunciamiento del Tribunal como en el anterior, se nota un doble enfoque sobre el ordenamiento comunitario andino, si bien el Tribunal sostiene la autonomía de las normas andinas al indicar que no depende de la de otros ordenamientos internacionales, ni debe sujetarse a que guarden compatibilidad o conformidad con ellas. Sin embargo en líneas siguientes, no deja de reconocer que las normas comunitarias andinas podrían estar inspiradas en el ordenamiento jurídico internacional.

Sobre la labor interpretativa de las normas andinas por parte del Tribunal de Justicia de la Comunidad Andina, es menester señalar que se puede desarrollar a través de un mecanismo que se conoce como la interpretación prejudicial ${ }^{27}$ del ordenamiento jurídico de la Comunidad Andina, para garantizar su aplicación uniforme en el territorio de los Países Miembros. Este es un proceso en el que hay un principio de colaboración entre el Juez comunitario y los jueces nacionales. En ese orden, los jueces nacionales de los países andinos pueden solicitar la interpretación al Tribunal de Justicia de la Comunidad Andina de las normas comunitarias que estén conociendo dentro de un proceso nacional. El propósito que se busca es obtener uniformidad en la interpretación de dichas normas en los Países Miembros.

\subsection{Sobre las restricciones y gravámenes al comercio}

La Secretaría General de la CAN tiene competencia para efectuar las investigaciones para determinar si la medida aplicada unilateralmente por un País Miembro de la Comunidad Andina, constituye un gravamen o restricción al comercio intrasubregional ${ }^{28}$. Esta investigación puede impulsarse a solicitud de un País Miembro, de un particular interesado, o de oficio ${ }^{29}$.

Cuando se presume la aplicación de una medida restrictiva al comercio intracomunitario andino, la Secretaría General deberá

26 Tribunalde Justicia de la Comunidad Andina, sentencia 7-AI-98 (acción de incumplimiento), de 21 de julio de 1999.

27 Ibídem.

28 Vale destacar que por gravamen se entiende a: Los derechos aduaneros y cualesquier otros recargos de efectos equivalentes, sean de carácter fiscal, monetario o cambiario, que incidan sobre las importaciones. No quedarán comprendidos en este concepto las tasas y recargos análogos cuando correspondan al costo aproximado de los servicios prestados. Mientras que por restricciones de todo orden, se refiere a cualquier medida de carácter administrativo, financiero o cambiario, mediante la cual un País Miembro impida o dificulte las importaciones, por decisión unilateral. Véase en: Artículo 73 del Acuerdo de Cartagena.

29 Artículo 74 del Acuerdo de Cartagena, y artículo 46 del Reglamento de Procedimientos Administrativos de la Secretaría General de la Comunidad Andina. 
realizar una investigación en el marco del procedimiento administrativo andino, donde intervendrán las partes involucradas y presentarán sus argumentos, y si como resultado de la misma, la Secretaría General considerare que la medida aplicada por un País Miembro cae en los supuestos de restricción o gravamen, deberá emitir un pronunciamiento a través de una Resolución, con la exposición de los motivos por los cuales la medida constituye un gravamen o restricción al comercio, y dependiendo de la gravedad o urgencia del caso deberá otorgar un plazo al País Miembro imputado -no mayor a un mes- para que retire la medida considerada infractora ${ }^{30}$.

La infracción al Programa de Liberación acordado en el Acuerdo de Cartagena es sancionado, en la medida que es uno de los objetivos básicos de ese proyecto de integración, a tal punto que el propio Acuerdo dispone que: Los Países Miembros se abstendrán de aplicar gravámenes $y$ de introducir restricciones de todo orden a las importaciones de bienes originarios de la Subregión ${ }^{31}$.

\section{SOBRE LAS SALVAGUARDIAS}

El Acuerdo de Cartagena contempla la posibilidad de que los Países Miembros de la CAN puedan adoptar las salvaguardias intracomunitarias, es decir, aquellas medidas aplicables por un País Miembro que tienen como efecto la restricción temporal autorizada de las importaciones originarias de otro País Miembro, bajo determinados supuestos y de manera provisional. Toda vez que, el principio general es la libre circulación de mercancías, no obstante, las salvaguardias son mecanismos que los acuerdos comerciales prevén en caso de urgencia, de forma tal de que la restricción que se imponga esté regulada y no dejarlo al arbitrio unilateral del país, bajo la creencia de que de esa forma se podría evitar males mayores.

Cabe señalar que las salvaguardias intracomunitarias ${ }^{32}$ previstas en el Acuerdo de Cartagena pueden ser de diferentes modalidades, como son: la salvaguardia por balanza de pagos (artículo 95), aquella derivada del cumplimiento del Programa de Liberación (artículo 96), la salvaguardia propiamente dicha aplicable a productos específicos (artículo 97), la salvaguardia por devaluación monetaria (artículo 98).

De los tipos de salvaguardias andinas mencionadas, aquí se pone el acento en la salvaguardia para productos específicos prevista en el artículo $97^{33}$ del Acuerdo de Cartagena debido a varias razones, por

30 Artículo 55 del Reglamento de Procedimientos Administrativos de la Secretaría General de la CAN.

31 Artículo 77 del Acuerdo de Cartagena.

32 Capítulo XI del Acuerdo de Cartagena.

33 Dicho artículo dispone que:

Cuando ocurran importaciones de productos originarios de la Subregión, en cantidades 
una parte, está vinculada al examen que se propone hacer a partir de la doctrina desarrollada por la Secretaría General y la jurisprudencia andina en esta materia, y por otra parte, las medidas que han aplicado los Países Miembros al comercio intracomunitario se concentran mayormente en ese tipo de salvaguardia ${ }^{34}$.

Por consiguiente, los casos que se proponen para ser examinados, surgen por la aplicación de una medida de salvaguardia multilateral estipulada en el Acuerdo sobre Salvaguardias de la Organización Mundial del Comercio (OMC), por parte de un País Miembro (Ecuador) dirigida en el primer caso, a las importaciones de cerámica plana y en el segundo caso, a los pisos de madera provenientes de todos los países, incluidos los andinos.

Los grupos regionales o multilaterales, contemplan la posibilidad de que los países que puedan aplicar de forma temporal y bajo ciertos supuestos justificados y regulados, medidas de carácter restrictivo al comercio, como por ejemplo, las medidas de salvaguardia, que serán objeto de análisis en este trabajo ${ }^{35}$.

o en condiciones tales que causen perturbaciones en la producción nacional de productos específicos de un País Miembro, éste podrá aplicar medidas correctivas, no discriminatorias, de carácter provisional, sujetas al posterior pronunciamiento de la Secretaría General.

El País Miembro que aplique las medidas correctivas, en un plazo no mayor de sesenta días, deberá comunicarlas a la Secretaría General y presentar un informe sobre los motivos en que fundamenta su aplicación. La Secretaría General, dentro de un plazo de sesenta días siguientes a la fecha de recepción del mencionado informe, verificará la perturbación y el origen de las importaciones causantes de la misma y emitirá su pronunciamiento, ya sea para suspender, modificar o autorizar dichas medidas, las que solamente podrán aplicarse a los productos del País Miembro donde se hubiere originado la perturbación. Las medidas correctivas que se apliquen deberán garantizar el acceso de un volumen de comercio no inferior al promedio de los tres últimos años.

34 Véase documento: "Modificación de los mecanismos de salvaguardia que afectan al comercio intracomunitario", SG/dt 297, 13 de junio de 2005, en: <www.comunidadandina. org $>$ [consultado 14/03/2016].

En cuanto a otros mecanismos previstos en el Acuerdo de Cartagena, que no han sido invocados en los últimos años, podemos mencionar el Artículo 96, el cual se incluyó en el Acuerdo de Cartagena para atender situaciones de perjuicios ocasionados por el incremento de importaciones provenientes de los Países Miembros durante el Programa de Liberación (periodo de desgravación arancelaria). La conformación de la Zona de Libre Comercio Andina desde 1993, hace inaplicable esta salvaguardia.

Otro mecanismo que ha dejado de invocarse es el que se refiere a medidas para corregir desequilibrios en la balanza de pagos (Artículo 95 del Acuerdo de Cartagena). Para este mecanismo la normativa andina señala que el País Miembro que adopte medidas de este tipo, podrá extenderlas a los socios andinos previa autorización de la Secretaría General. Esta condición de autorización para la extensión de la medida, aunada al hecho que los países, rara o ninguna vez han recurrido a medidas de alivio comercial de tipo general para corregir desequilibrios de balanzas de pagos, ha hecho estricta e inaplicable esta salvaguardia en el ámbito intracomunitario. p.4.

Según el estudio de la Secretaría General, entre 1990 a mayo de 2005, las solicitudes realizadas por los Países Miembros se concentran en el mecanismo para productos específicos previsto en el Artículo 97 del Acuerdo de Cartagena (68 por ciento de las solicitudes) Ibídem.

35 Véase por ejemplo, en el caso de la integración regional andina, el Capítulo XI del Acuerdo de Cartagena, que contiene las Cláusulas de Salvaguardia; y por otra parte, en el caso del sistema multilateral del comercio, el Acuerdo sobre Salvaguardias de la Organización 
Ahora bien, como se ha explicado, ocurre que los países pueden formar parte de un acuerdo regional de integración y de un acuerdo multilateral como la OMC, en ese sentido, dispondrían del acceso a dos mecanismos de salvaguardias, por un parte, la salvaguardia regional, y por otra parte, la salvaguardia de la OMC, ambas medidas reguladas por normas propias y con una alcance dirigido a sus miembros.

Es preciso advertir que el Tribunal Andino de Justicia ha señalado que las medidas de salvaguardia constituyen un remedio extremo que sólo se permite por vía de excepción, como defensa necesaria, aunque transitoria, de los países comprometidos en el proceso de integración, ante trastornos graves e imprevistos ${ }^{36}$. Por su parte, el Órgano de Apelación de la Organización Mundial del Comercio ha expresado que las medidas de salvaguardias son medidas correctivas de carácter extraordinario que sólo pueden adoptarse en situaciones de urgencia ${ }^{37}$.

Como puede observarse, ambas instancias coinciden en reconocer el carácter excepcional de estas medidas, que estarían permitidas ante situaciones graves o de urgencia, y a través de ellas se buscaría entonces, proveer una protección temporal a la rama de la producción nacional afectada.

En el razonamiento que plantea el Tribunal Andino para justificar las salvaguardias, subsiste la preocupación que:

De no existir esta previsión, llevarían presumiblemente a una situación insostenible para el país afectado, con la lógica consecuencia de incumplimientos forzosos e inevitables del programa de liberación, o aun de francos rompimientos del propio acuerdo, que sin duda afectaría más seriamente el proceso de integración que el uso regulado y controlado de la salvaguardia, la que actúa así, como un medio para evitar males mayores ${ }^{38}$.

Esto es, se pretende corregir un desequilibrio con la aplicación temporal de una salvaguardia comercial regulada por el propio organismo comunitario, de forma de garantizar que no se cometan excesos que puedan afectar la libertad de comercio entre los Países Miembros, que es el fin último que debe prevalecer. En ese orden, el Tribunal Andino señala que:

Mundial del Comercio. En ese orden, vale señalar que en el modelo andino, en materia de comercio de bienes, los países miembros disponen de la posibilidad de aplicar medidas de salvaguardia contenidas en el Acuerdo de Cartagena, para el comercio andino, son las llamadas salvaguardias intracomunitarias. Ibídem.

36 Sentencia de 10 de junio de 1987, proceso 1-N-86, p.69.

37 Ver por ejemplo: "Medidas de salvaguardia definitiva contra las importaciones de tubos de carbono soldados de sección circular procedentes de Corea", WT/DS202/R, 15 de febrero de 2002, párrafo 80. En: <https://www.wto.org/> [consultado 23/04/2016].

38 En sentencia del Tribunal de Justicia Andino de 10 de junio de 1987, proceso 1-N-86 (acción de nulidad). 
Debe evitarse que estas situaciones excepcionales hagan imposible el proceso de integración, o que lo interrumpan o retrasen más allá de lo estrictamente necesario. La debida conciliación de estos intereses, los del país afectado y los de la integración, habrá de ser entonces criterio básico para la interpretación y aplicación de las normas del Acuerdo ${ }^{39}$.

Por su parte en el espacio multilateral, la salvaguardia consiste en un mecanismo mediante el cual, un miembro de la Organización Mundial del Comercio puede restringir de forma temporal las importaciones de un producto, si las importaciones de ese producto han aumentado en tal cantidad que, causan o amenazan causar daño a una rama de producción nacional ${ }^{40}$.

De lo expuesto anteriormente se desprenden las características de las medidas de salvaguardia, a saber: a) carácter extraordinario; y b) su aplicación temporal. Un país como miembro de ambos esquemas de comercio, podría interpretar que puede escoger entre una u otra medida, y aplicarla al comercio que considere afectado. Pero como se mostrará, esa doble regulación en la materia descrita, así como, la vinculación de los países a un acuerdo de integración y a un acuerdo multilateral de comercio, ha dado lugar a que surja una interpretación del derecho comunitario andino, sobre el alcance de la aplicación de las normas regionales y multilaterales en materia de salvaguardias a los países de la Comunidad Andina.

\section{LA JURISPRUDENCIA COMUNITARIA ANDINA: ANÁLISIS DE CASOS SOBRE LA RELACIÓN ENTRE LA MEDIDA DE SALVAGUARDIA REGIONAL ANDINA Y LA SALVAGUARDIA MULTILATERAL DE LA OMC}

En este apartado se expondrán los principales aspectos de dos controversias suscitadas entre Países Miembros de la Comunidad Andina donde se debatía sobre la aplicabilidad de una norma regional andina en materia de salvaguardia comercial, prevista en el Acuerdo de Cartagena y, por otra parte, sobre una norma multilateral de la Organización Mundial del Comercio, como es el Acuerdo sobre Salvaguardias de la OMC.

El primer planteamiento se inició en la Secretaría General de la Comunidad Andina, con una investigación que tuvo por objeto determinar la posible aplicación de gravámenes por parte de Ecuador a las importaciones de un producto, cerámica plana (subpartidas NANDINA 6908.90.00 y 6907.90.00), originarias de Países Miembros de la Comunidad Andina.

Ecuador adoptó dos Resoluciones, primero, la Resolución 205 del

39 Ibídem.

40 Véase Acuerdo sobre Salvaguardias de la OMC, artículo 2. 
Consejo de Comercio Exterior e Inversiones del Ecuador (COMEXI), publicada en el Registro Oficial del Ecuador 159 de 1 de septiembre de 2003, con el objetivo de:

Artículo 1.- Aplicar una medida de salvaguardia provisional, consistente en un derecho adicional del 15 por ciento ad-valórem, al arancel nacional vigente, que al momento es del 15 por ciento, para las importaciones de cerámica plana clasificadas en la subpartida NANDINA 6908.90.00 del arancel nacional, provenientes de todos los países; es decir un total del 30 por ciento, de conformidad con lo que establece el artículo 6 del Acuerdo sobre Salvaguardias de la Organización Mundial del Comercio ${ }^{41}$ y el artículo 322 del texto unificado de Legislación del MICIP.

Posteriormente, Ecuador emitió la Resolución 232 del COMEXI, publicada en el Registro oficial 273 de 13 de febrero de 2004, mediante la cual decidió:

Artículo 1.- Aplicar una medida de salvaguardia definitiva, por un período de dieciocho meses calendario, consistente en el establecimiento de un derecho específico adicional al arancel vigente para las importaciones de cerámica plana, clasificadas en las subpartidas NANDINA 6908.90.00 y 6907.90.00 del Arancel Nacional, provenientes de todos los países, de conformidad con lo que establecen los artículos 3.1 y 5.1 del Acuerdo sobre Salvaguardias de la Organización Mundial del Comercio ${ }^{42}$; y los

41 Véase artículo 6: Medidas de salvaguardia provisionales: En circunstancias críticas, en las que cualquier demora entrañaría un perjuicio difícilmente reparable, un Miembro podrá adoptar una medida de salvaguardia provisional en virtud de una determinación preliminar de la existencia de pruebas claras de que el aumento de las importaciones ha causado o amenaza causar un daño grave. La duración de la medida provisional no excederá de 200 días, y durante ese período se cumplirán las prescripciones pertinentes de los artículos 2 a 7 y 12. Las medidas de esa índole deberán adoptar la forma de incrementos de los aranceles, que se reembolsarán con prontitud si en la investigación posterior a que se refiere el párrafo 2 del artículo 4 no se determina que el aumento de las importaciones ha causado o amenazado causar un daño grave a una rama de producción nacional. Se computará como parte del período inicial y de las prórrogas del mismo a que se hace referencia en los párrafos 1,2 y 3 del artículo 7 la duración de esas medidas provisionales.

42 Véase Acuerdo sobre Salvaguardias, OMC: Artículo 3: Investigación: 1. Un Miembro sólo podrá aplicar una medida de salvaguardia después de una investigación realizada por las autoridades competentes de ese Miembro con arreglo a un procedimiento previamente establecido y hecho público en consonancia con el artículo X del GATT de 1994. Dicha investigación comportará un aviso público razonable a todas las partes interesadas, así como audiencias públicas u otros medios apropiados en que los importadores, exportadores y demás partes interesadas puedan presentar pruebas y exponer sus opiniones y tengan la oportunidad de responder a las comunicaciones de otras partes y de presentar sus opiniones, entre otras cosas, sobre si la aplicación de la medida de salvaguardia sería o no de interés público. Las autoridades competentes publicarán un informe en el que se enuncien las constataciones y las conclusiones fundamentadas a que hayan llegado sobre todas las cuestiones pertinentes de hecho y de derecho.

Artículo 5: Aplicación de medidas de salvaguardia:

1. Un Miembro sólo aplicará medidas de salvaguardia en la medida necesaria para prevenir 
artículos 318 y 326 del Texto Unificado de Legislación del MICIP.

La Resolución 232 del Ecuador dispuso que:

Artículo 5.- Excluir de la medida de salvaguardia definitiva a los países en desarrollo que, de conformidad con lo que establece el artículo 9.1 del Acuerdo sobre Salvaguardias de la $\mathrm{OMC}^{43}$, registren importaciones inferiores al 3 por ciento del total de importaciones anuales, a condición de que el porcentaje de esos países no represente, en conjunto, más del 9 por ciento de las importaciones totales. Los países en desarrollo que al momento registran importaciones y cumplen con la condición señalada en el párrafo anterior son: Venezuela, Emiratos Árabes Unidos, Indonesia, Corea del Sur, Hong Kong, Malasia, India, Chile, Argentina, México y Viet Nam.

Sobre la prohibición de discriminar, Ecuador sostenía que, según el artículo 2.2 del Acuerdo sobre Salvaguardias de la OMC, las medidas de salvaguardia se aplicarán al producto importado independientemente de la fuente de donde proceda ${ }^{44}$. En ese sentido, proseguían con el argumento que discriminar el origen de los productos, excluyendo de la aplicación de la salvaguardia a los productos originarios de la Comunidad Andina de Naciones, significaría poner en riesgo la validez del proceso y la validez de las medidas ante la Organización Mundial del Comercio $^{45}$. Por su parte, la Secretaría General de la Comunidad Andina observó que las normas de la OMC permiten a los Países Miembros de Acuerdos Regionales de Comercio excluir entre ellos la aplicación de medidas de salvaguardia previstas en las normas de la $\mathrm{OMC}^{46}$.

Como parte en el proceso, Colombia sostuvo a semejanza de lo expuesto por la Secretaría General, que las salvaguardias aplicables al comercio entre los miembros de la CAN, se rigen por el Acuerdo de Cartagena y demás reglamentaciones de su ordenamiento jurídico ${ }^{47}$.

o reparar el daño grave y facilitar el reajuste. Si se utiliza una restricción cuantitativa, esta medida no reducirá la cuantía de las importaciones por debajo del nivel de un período reciente, que será el promedio de las importaciones realizadas en los tres últimos años representativos sobre los cuales se disponga de estadísticas, a menos que se dé una justificación clara de la necesidad de fijar un nivel diferente para prevenir o reparar el daño grave. Los Miembros deberán elegir las medidas más adecuadas para el logro de estos objetivos.

43 Artículo 9:Países en desarrollo Miembros:

1. No se aplicarán medidas de salvaguardia contra un producto originario de un país en desarrollo Miembro cuando la parte que corresponda a éste en las importaciones realizadas por el Miembro importador del producto considerado no exceda del 3 por ciento, a condición de que los países en desarrollo Miembros con una participación en las importaciones menor del 3 por ciento no representen en conjunto más del 9 por ciento de las importaciones totales del producto en cuestión.

44 En la Resolución 838 de la Secretaría General, de 16 de julio de 2004. En: <www. comunidadandina.org/normativa/> [consultado 12/05/2016].

45 Ibídem.

46 Ibídem.

47 Ibídem. 
La adopción de las medidas señaladas por Ecuador motivó el inicio de una investigación en la Secretaría General por posible aplicación de gravamen al comercio andino, con base en el artículo 30 literal a) y el Capítulo VI del Acuerdo de Cartagena sobre el Programa de Liberación, y el Reglamento de Procedimientos Administrativos de la Secretaría General, y concluyó que las medidas exigidas por Ecuador -derechos ad valorem y derechos específicos- a las importaciones de cerámica plana provenientes de los Países Miembros de la Comunidad Andina, a través de las Resoluciones 205 y 232 del Consejo de Comercio Exterior e Inversiones del Ecuador, constituían un gravamen al comercio intrasubregional andino ${ }^{48}$.

Ante el argumento de Ecuador en el sentido de poder aplicar las medidas de salvaguardia del Acuerdo sobre Salvaguardias, a todos los países, incluso a los Países Miembros de la Comunidad Andina ${ }^{49}$, la Secretaría General se pronunció sobre la relación entre las salvaguardias de la OMC y la prohibición de aplicar gravámenes al comercio intracomunitario, y sostuvo, con base en la jurisprudencia del Tribunal de Justicia de la Comunidad Andina que:

La circunstancia de que los Países Miembros de la Comunidad Andina pertenezcan a su vez a la Organización Mundial de Comercio no los exime de obedecer las normas comunitarias andinas so pretexto de que se está cumpliendo con las de dicha organización o que se pretende cumplir con los compromisos adquiridos con ella ${ }^{50}$.

La Secretaría General de la Comunidad Andina también señaló que el artículo XIX del GATT y el Acuerdo de Salvaguardias de la OMC no imponen a sus Miembros una obligación de aplicar medidas de salvaguardia. Esta es una facultad que disponen los Miembros de la $\mathrm{OMC}$, cuyo ejercicio no justifica la adopción de medidas incompatibles con otros compromisos que aquellos hubieran asumido en el marco de acuerdos regionales ${ }^{51}$.

Según lo expresado por la Secretaría General, entonces, los Países Miembros de la Comunidad Andina sólo habrían podido aplicar gravámenes al comercio intracomunitario, amparándose en las normas

48 Véase la Resolución 814 de la Secretaría General de la Comunidad Andina, de 7 de abril de 2004, publicada en la Gaceta Oficial del Acuerdo de Cartagena 1052 de 12 de abril del mismo año. En dicha Resolución, la Secretaría General, otorgó a Ecuador un plazo de diez días calendario para el levantamiento del gravamen a las importaciones andinas correspondientes. Esta Resolución está disponible en la página web de la Comunidad Andina: <www. comunidadandina.org/normativa/> [14/05/2016] seleccionar Resolución 814.

49 Cabe destacar que Ecuador excluyó a Venezuela de la aplicación de la salvaguardia con fundamento en el artículo 9.1 del Acuerdo sobre Salvaguardias.

50 Sentencia del Tribunal de Justicia de la Comunidad Andina, 21 de julio de 1999, en el proceso 7-AI-98.

51 Ibídem. Resolución 814. 
de la OMC, si el Acuerdo de Cartagena expresamente hubiera previsto esa posibilidad ${ }^{52}$. Y en ese sentido de argumentación de la Comunidad Andina, las medidas de salvaguardia al comercio intracomunitario andino aplicables por los andinos serían las pautadas en el Acuerdo de Cartagena, en su Capítulo XI ${ }^{53}$.

El criterio expuesto fue ratificado posteriormente por dicho órgano comunitario en la Resolución $838^{54}$ de 16 de julio de 2004, que declaró sin lugar el recurso de reconsideración interpuesto por Ecuador contra la Resolución 814 del 7 de abril de 2004.

Es preciso señalar que con ocasión del pronunciamiento de la Secretaría General de la Comunidad Andina, en la fase administrativa (pre contenciosa) ${ }^{55}$, Ecuador acató lo dispuesto por dicho órgano comunitario $^{56}$, y procedió a levantar la medida de salvaguardia examinada, pero sólo a las importaciones de los países de la Comunidad Andina.

En el segundo planteamiento que se expondrá a continuación, la Secretaría General de la CAN ratificó la jurisprudencia del Tribunal de Justicia de la CAN desarrollada en el caso precedentemente expuesto, y sentó su posición en la Resolución 1796 del 24 de julio de 2015, publicada en la Gaceta Oficial del Acuerdo de Cartagena N²538 de la misma fecha.

En esta oportunidad, la Secretaría General resolvió la solicitud presentada por Perú, mediante el Oficio N ${ }^{\circ}$ 26-2015-MINCETUR/ VMCE, del 21 de enero de 2015, para que se calificara como gravamen a la medida de salvaguardia provisional aplicada por Ecuador mediante la Resolución 001-2014, a través del procedimiento de investigación por salvaguardias iniciada en el marco de la OMC, por el gobierno del Ecuador mediante Resolución 0262014 del COMEX.

En el petitorio Perú exigía Ecuador levantar la medida de salvaguardia provisional impuesta a los productos de las subpartidas arancelarias NANDINA 44091010 (tablillas y frisos para parqués, sin ensamblar);

\section{Ibídem.}

53 El Acuerdo de Cartagena no establece la posibilidad de que los países Miembros apliquen otro tipo de medidas de salvaguardia, diferentes a las previstas en el Acuerdo. Por lo que cualquier aplicación de medidas de salvaguardia al comercio intracomunitario se regirá bajo lo dispuesto en el ordenamiento jurídico de la Comunidad Andina, en: Resolución 838 de la Secretaría General.

54 La Secretaría General de la Comunidad Andina, señaló en la Resolución 838, que el recurso de reconsideración interpuesto por los recurrentes no desvirtuaron los criterios expuestos precedentemente, y declaró sin lugar el recurso de reconsideración interpuesto contra la Resolución 814 de la Secretaría General.

55 Contenidas en las Resoluciones 814 y 838.

56 Puede revisarse: el documento: "Examen de políticas comerciales", WT/TPR/S/148, p.87 en: <www.mmrree.gov.ec/mre/documentos/pol_internacional/multilateral/OMC/> [8/04/2016]; véase también: <http://www.aduana.gov.ec/>, Boletín de la Corporación Aduanera Ecuatoriana, de 14 de mayo de 2004. El estado de cumplimiento de la norma andina por parte de Ecuador puede verificarse en: Informe de la Secretaría General sobre cumplimiento de compromisos y solución de controversias de 26 de enero de 2006. SG/di 776, en: <http:// intranet.comunidadandina.org/IDocumentos/> [8/04/2016]. 
44091020 (madera moldurada); 44091090 (las demás); 44092100 (de bambú); 4409291 (tablillas y frisos para parqués, sin ensamblar); 44092920 (madera moldurada); 44092990 (las demás), especificadas en el Oficio $\mathrm{N}^{\circ}$ 84-2015-MINCETUR/VMCE del 6 de marzo de 2015.

La Secretaría General de la Comunidad Andina admitió el 7 de mayo de 2015, la solicitud sobre calificación como gravamen de la salvaguardia OMC aplicada a los pisos de madera y de bambú y sus accesorios por parte del Ecuador a los países andinos, dando inicio al procedimiento de conformidad con lo dispuesto en los artículos 49, 50 y 51 de la Decisión 425.

Ahora bien, al respecto, Ecuador solicitó que el caso se archivara debido a que mediante Resolución 022-2015 del COMEX, en vigencia desde el 23 de mayo de 2015:

Se adoptó una medida de salvaguardia definitiva en el marco de la Organización Mundial del Comercio (OMC), a las importaciones de pisos de madera y de bambú y a sus accesorios, resultando excluida la República del Perú, entre otros países en desarrollo, ya que sus importaciones alcanzaron el nivel de minimis establecido en el artículo 9 del Acuerdo de Salvaguardias de la OMC.

La medida consistía en una sobretasa arancelaria adicional, no discriminatoria, al arancel general, a productos clasificados en las subpartidas arancelarias mencionadas. Pero, Ecuador alegaba que no existía, desde el 23 de mayo de 2015, la supuesta restricción alegada por Perú.

Sin embargo, cabe observar que la solicitud de Perú buscaba la calificación de gravamen de la medida (salvaguardia provisional) aplicada por Ecuador mediante la Resolución 001-2014 del Ministerio de Comercio Exterior, en los siguientes términos:

Se debe determinar la exclusión del Perú de la investigación iniciada por Ecuador bajo la normativa de la OMC, dado que un País Miembro de la CAN solo puede imponer medidas de salvaguardia a las importaciones originarias de los demás Países Miembros en estricta observancia de la normativa comunitaria sobre la materia, no pudiendo aplicar para tal efecto la normativa de la OMC. Si una misma materia está regulada en la CAN y en la OMC, los Países Miembros comunitarios deben aplicar la norma emitida por sus respectivas organizaciones para sus relaciones intracomunitarias.

Se debe disponer que Ecuador levante la medida impuesta el 23 de octubre de 2014, contra las importaciones de origen peruano de pisos de madera y de bambú, por devenir en irregular, al no haber efectuado la notificación correspondiente ni presentada el informe que la justifique a la SGCAN. 
Según lo expuesto, si bien es cierto que, durante el procedimiento administrativo iniciado en la Secretaría General de la Comunidad Andina, la medida (salvaguardia provisional) aplicada por Ecuador mediante la Resolución 001-2014 del Ministerio de Comercio Exterior, dejó de aplicarse a partir del 23 de mayo de 2015, y también que, dicha medida se transformó en definitiva, según la Resolución Nº 022-2015 del COMEX, y excluyó de su aplicación a diversos países, entre los que se encuentra Perú. Sin embargo, la Secretaría General de la CAN observó que en el lapso comprendido entre la fecha de aplicación de le medida reclamada (provisional) y su derogatoria, se cobró una sobretasa al arancel general vigente de USD 1.50 por kilogramo importado independientemente del origen, a las importaciones de productos clasificados en las subpartidas arancelarias NANDINA señaladas mediante la Resolución 001-2014 del Ministerio de Comercio Exterior del Ecuador.

Por lo cual, la Secretaría General de la CAN advirtió en la Resolución 1796 que la aplicación de esa medida no autorizada la convierte en una medida de facto y, por ende, en un gravamen a la luz de lo dispuesto en el artículo 73 del Acuerdo de Cartagena. A su vez, la Secretaría General consideró en dicha Resolución que:

Lo solicitado por el gobierno del Perú referido a que se califique como gravamen, se levante la medida dispuesta mediante la Resolución 001-2014 del Ministerio de Comercio Exterior y se le excluya del procedimiento de investigación por salvaguardias iniciada en el marco de la OMC, por el gobierno del Ecuador mediante Resolución 026-2014 del COMEX, se encuentra cumplido con lo dispuesto en la Resolución $\mathrm{N}^{\circ}$ 022-2015 del COMEX, por lo que en aplicación de los principios de economía procesal y eficacia de los procedimientos, señalados en el artículo 5 de la Decisión 425; corresponde dar por terminado el presente trámite por sustracción de materia.

En este caso, la Secretaría General fue consecuente con la jurisprudencia comunitaria andina y recordó la obligación de los Países Miembros de cumplir lo señalado en el Capítulo IX de Acuerdo de Cartagena y en particular su artículo 97, que ordena que las medidas correctivas deben comunicarse a la Secretaría General y justificarse ante ella, con la finalidad de que ésta verifique su procedencia y suspenderla, modificarla o autorizarla. El cumplimiento de esas obligaciones es independiente de cualquier actuación que los Países Miembros realicen en cualquier otro foro, incluso el multilateral.

En ese orden de ideas, no podría justificarse el patrocinio de medidas de salvaguardia como la analizada. Por consiguiente, la Secretaría General de la CAN ratificó en la Resolución 1796 que:

Ello sería ni más ni menos que negar la supremacía del ordenamiento comunitario andino que como se ha dicho es preponderante no sólo respecto 
de los ordenamientos jurídicos internos de los Países Miembros sino de los otros ordenamientos jurídicos internacionales a que éstos pertenezcan. Al respecto, la jurisprudencia de este Tribunal ha dejado claramente expresada la naturaleza del principio de supremacía del derecho comunitario ${ }^{57}$.

\section{CONCLUSIÓN}

La doble vinculación de los países a normas regionales y multilaterales, es susceptible de interpretaciones de distinto alcance. Por una parte, un país andino (Ecuador) invocó en dos ocasiones, la aplicación de una medida de salvaguardia multilateral contenida en el Acuerdo sobre Salvaguardias de la OMC, sobre las importaciones de todos los países, eso incluía a países andinos. Por otra parte, la argumentación de la instancia pre contenciosa andina a través de Secretaría General, así como la jurisprudencia del Tribunal Andino de Justicia, ha sido consecuente en sostener que la aplicación de medidas de salvaguardia al comercio intracomunitario se regirá bajo lo dispuesto en el ordenamiento jurídico de la Comunidad Andina, cuando se den las causales que justifiquen su adopción. Dichas medidas están sujetas a la autorización o no de la Secretaría General de la Comunidad Andina, debido a que es una competencia específica que fue atribuida por los países andinos a ese órgano comunitario, en ese sentido, también fue la interpretación de otros países andinos (Colombia y Perú).

Por otra parte, los países andinos tienen a su disposición la posibilidad de aplicar la medida de salvaguardia contenida en el Acuerdo sobre Salvaguardias de la OMC, bajo los supuestos contemplados en dichas normas.

Es por ello que, la labor interpretativa de la Secretaría General y del Tribunal de Justicia de la Comunidad Andina, ha contribuido a reafirmar la uniformidad y autonomía de las normas andinas y a salvaguardar los objetivos propuestos en el Acuerdo de Cartagena.

\section{REFERENCIAS BIBLIOGRÁFICAS}

COMISIÓN DE LA COMUNIDAD ANDINA. Codificación del Acuerdo de Integración Subregional Andino (Acuerdo de Cartagena). Decisión 563. Lima. 26 de junio de 2003. [consulta 8/05/2016]. Disponible en: $<$ http://www.comunidadandina.org/normativa/>

COMISIÓN DE LA COMUNIDAD ANDINA. Tratado de Creación del Tribunal de Justicia de la Comunidad Andina. Decisión 472. Cochabamba. 28 de mayo de 1996. [consulta $16 / 04 / 2016$ ]. Disponible en: <http:// www.comunidadandina.org/normativa/>

$\overline{57 \text { Proceso } \mathrm{N}^{\circ} 01-\mathrm{AI}}-2001$ 
COMISIÓN DE LA COMUNIDAD ANDINA. Reglamento de Procedimientos Administrativos de la Secretaría General de la Comunidad Andina. Decisión 425. Montevideo. 14 de diciembre de 1997. [consulta 12 /06/2016]. Disponible en: <http://www.comunidadandina.org/ normativa/>

CONSEJO ANDINODEMINISTROSDERELACIONESEXTERIORES. Estatuto del Tribunal de Justicia de la Comunidad Andina. Decisión 500. Valencia. 22 de junio de 2001. [consulta 16/06/2016]. Disponible en: $<$ http://www.comunidadandina.org/normativa/>

FERRER, Aldo. "Desarrollo e Integración: Desafíos y Oportunidades del MERCOSUR, Parte I y II". Revista Vértice. Caracas. 2007, n², p. 7-9.

KEOHANE, Robert O., NYE, Joseph. Poder e Interdependencia. Buenos Aires. Grupo Editor Latinoamericano. Colección Estudios Internacionales, 1988.

LEVI, Michel. "La Comunidad Andina y el referente dela Unión Europea: Una visión temporal sobre la aplicación de los modelos de integración". La Unión Europea y la Integración regional. Perspectivas comparadas y lecciones para las Américas, Buenos Aires: CARI, Eduntref, University of Miami, 2005, p.267-295.

MALDONADO LIRA, Héctor. 30 años de Integración andina, Balances y Perspectivas. Lima: Secretaría General de la Comunidad Andina, 1999.

MONSANTO, Alberto. "CAN-MERCOSUR: Esquemas institucionales comparados". En: UNIVERSIDAD NACIONAL DEL ROSARIO, CÁTEDRA INTERNACIONAL ANDRÉS BELLO (ed.). Integración y Cooperación Atlántico Pacífico. Rosario: Editorial Universidad Nacional de Rosario, 2002.

NARANJO Fernando. "Política Exterior e Integración. Oportunidades y Desafíos". En: Cuadernos Integración en America Latina. Santiago de Chile: CEPAL/Fundación Carolina/FLACSO, 2006.

ORGANIZACIÓN MUNDIAL DEL COMERCIO. Acuerdo sobre Salvaguardias de la OMC. [consulta 13/10/2015]. Disponible en: $<$ http:// www.wto.org>

ORGANIZACIÓN MUNDIAL DEL COMERCIO. Medidas de salvaguardia definitiva contra las importaciones de tubos de carbono soldados de sección circular procedentes de Corea, WT/DS202/R, 15 de febrero de 2002, párrafo 80. [28/04/2016]. En: https://www.wto.org/ (23/04/2016); "Examen de políticas comerciales", WT/TPR/S/148, p.87. Disponible en: <www.mmrree.gov.ec/mre/documentos/pol_ internacional/multilateral/OMC/>

SECRETARÍA GENERAL DE LA COMUNIDAD ANDINA. Resoluciones No 814 de 7 de abril de 2004; No838 de 16 de julio de 2004; y $N^{\circ} 1796$ de 24 de julio de 2015. [consulta 20/11/2015]. Disponible en: <http://www.comunidadandina.org/canprocedimientosinternet/ procedimientos.aspx $>$ 
SECRETARÍA GENERAL DE LA COMUNIDAD ANDINA. Modificación de los mecanismos de salvaguardia que afectan al comercio intracomunitario", SG/dt 297, Lima, 13 de junio de 2005. [consulta 12/04/2016)]. Disponible en: <http://www.comunidadandina.org/ canprocedimientosinternet/procedimientos.aspx >

SESELOVSKY, Ernesto, et al. "Necesidad de armonización de las políticas macroeconómicasenlosprocesosdeintegraciónregional".En:UNIVERSIDAD NACIONAL DEL ROSARIO, CÁTEDRA INTERNACIONAL ANDRÉS BELLO (ed.). Integración y Cooperación Atlántico-Pacífico. Rosario: Editorial Universidad Nacional de Rosario, 2002.

SUNKEL, Osvaldo. "Reflexiones sobre América Latina y el Caribe. Desarrollo e Integración Regional”. Revista de la CEPAL. 1998, número extraordinario.

TRIBUNAL DE JUSTICIA DE LA COMUNIDAD ANDINA. Testimonio Comunitario. CD-168. Quito. Tribunal de Justicia de la Comunidad Andina. 2004.

TRIBUNAL DE JUSTICIA DE LA COMUNIDAD ANDINA. Sentencia 1-IP-96 (interpretación prejudicial); sentencia 2 IP 88, sentencias 7-AI-98 y 89-AI-2000 (acción de incumplimiento), sentencias 1-N-86, 10 AN 2000 y 61 AN 2000 (acción de nulidad). [consultas 8/11/2015 y 16/06/2016]. Disponible en: <http://www.comunidadandina.org/ canprocedimientosinternet/procedimientos.aspx $>$ 